\title{
Dolor inguinal en el fútbol. Factores de riesgo y estrategias metodológicas de intervención: prevención, rehabilitación y readaptación físico-deportiva. Revisión bibliográfica
}

\author{
Groin pain in soccer. Risk factors and methodological strategies of intervention: \\ prevention, rehabilitation and physical-sports readaptation. Bibliographic review
}

\author{
Iván Asín-Izquierdo ${ }^{1,4}$, Alberto Arribas-Romano ${ }^{2}$, Marcos Chena ${ }^{1,5}$, David García-Herrero ${ }^{1}$, \\ Luis Gutiérrez-García ${ }^{1,6}$, Marcos José Navarro-Santana ${ }^{3}$ \\ 1 Departamento de Ciencias Biomédicas, Facultad de Medicina y Ciencias de la Salud, Universidad de Alcalá, Madrid, España. \\ 2 Departamento de Fisioterapia, Terapia Ocupacional, Rehabilitación y Medicina Física, Universidad Rey Juan Carlos, Alcorcón, España. \\ 3 Centro Médico Rehabilitación San Fernando, Madrid, España \\ 4 Atlético Astorga F.C. \\ 5 Real Racing Club de Santander. \\ 6 A.D. Torrejón C.F.
}

CORRESPONDENCIA:

Marcos José Navarro-Santana

marcosjose.navarrosantana@gmail.com
CÓMO CITAR EL ARTÍCULO:

Asín-Izquierdo, I., Arribas-Romano, A., Chena, M., García-Herrero, D., Gutiérrez-García, L., \& Navarro-Santana, M. J. (2020). Dolor inguinal en el fútbol. Factores de riesgo y estrategias metodológicas de intervención: prevención, rehabilitación y readaptación físico-deportiva. Revisión bibliográfica. Cultura, Ciencia y Deporte, 15(44), 255-266.

\section{Abstract}

El dolor inguinal referido al deporte es una patología muy compleja, por ello es fundamental conocer la lesión en profundidad para poder realizar una intervención adecuada, determinando los elementos necesarios para desarrollar un plan preventivo o recuperación / readaptación físico-deportiva, teniendo en cuenta la complejidad y la individualización de la patología. En este estudio se realiza una revisión de los diferentes factores de riesgo de dolor inguinal en fútbol y de las propuestas metodológicas de intervención en prevención, recuperación y readaptación físico-deportiva, determinando unas bases fundamentales en el abordaje del dolor inguinal referido al deporte en fútbol.

Palabras clave: dolor inguinal, lesión, fútbol, readaptación, prevención.

\begin{abstract}
The groin pain referred to sports is a very complex pathology, so it essential to know the injury in depth to be able to perform an appropriate intervention, determining the most important elements which are necessary to develop on a preventive plan or recovery / physical-sport rehabilitation, having in consideration the complexity and the individualization of each case of the pathology. In this study, a review is made of the different risk factors of groin pain in soccer and of the methodological proposals for intervention in prevention, recovery and physical-sport rehabilitation, determining fundamental bases in the approach to groin pain referred to sport of soccer.
\end{abstract}

Key words: groin pain, injury, soccer, readaptation, prevention. 


\section{Introducción}

En el fútbol, debido a la tipología de esfuerzos y características del entrenamiento y del deporte en sí, determinadas lesiones son muy habituales, ya sea en el contexto del entrenamiento o la competición. El dolor inguinal, junto con el esguince de tobillo, las lesiones de rodilla, lesiones musculares, tendinopatías y lumbalgias son las lesiones más comunes en futbolistas (Hägglund, Waldén, \& Ekstrand, 2005; Holmich, Thorborg, Dehlendorff, Krogsgaard, \& Gluud, 2014; Panasiuk, 2009; Waldén, Hagglund, \& Ekstrand, 2005).

La incidencia de lesiones en fútbol profesional, a nivel general, es de 8.9 lesiones (l)/1000 horas (h) de práctica deportiva, cifra que se eleva observando datos en competición, llegando incluso a 41.3 1/1000h (Noya \& Sillero, 2012b, 2012a). Aunque otros estudios establecen cifras más reducidas, como Holmich et al. (2014) con 3.41/1000h en fútbol semiprofesional, el rango normal, en los resultados de los estudios en fútbol profesional, es de entre 6 a 9l/1000h (Ekstrand, Hägglund, \& Waldén, 2011b; Salces et al., 2014) con tasa de recidiva del $10-12 \%$ o incluso de 13-22\% (Ekstrand, Hägglund, \& Waldén, 2011a). La incidencia lesional en fútbol amateur es más reducida, pero con mayor recurrencia que la de fútbol profesional, con $5.2 \mathrm{l} / 1000 \mathrm{~h}$ y $35.1 \%$ de tasa de recurrencia lesional, frente a 7.2-7.41/1000h y 16.6-25\% en fútbol de máximo nivel y élite, además conforme aumenta el nivel de rendimiento aumentan las lesiones totales y la diferencia entre lesiones en entrenamiento y competición (Hägglund, Waldén, \& Ekstrand, 2016). La demarcación no es un determinante claro en la incidencia lesional, existiendo cierta controversia; se observa una tendencia de los porteros a sufrir menos lesiones en comparación con los jugadores de campo y de los delanteros a sufrir más lesiones en la competición (Della-Villa, Mandelbaum, \& Lemak, 2018). La mayoría de las lesiones son ocasionadas durante el partido, siendo el $21 \%$ causadas por juego sucio y con una incidencia creciente con el paso de los minutos en la primera y segunda parte del partido (Ekstrand, Hägglund, \& Waldén, 2011b). Esta alta incidencia de las lesiones en el deporte provoca un alto impacto socioeconómico (Polinder, Haagsma, Panneman, Scholten, Brugmans, \& Van Beeck, 2016). Afortunadamente, la mayoría de las lesiones suelen ser de mínima gravedad (1-3 días perdidos por el deporte) (Ekstrand, Hägglund, \& Waldén, 2011a). Los programas de prevención en el fútbol han demostrado, mediante evidencia empírica, que pueden reducir el riesgo de lesiones a nivel general, ya sean lesiones musculares, ligamentosas, tendinosas y articulares, (Al Attar,
Soomro, Pappas, Sinclair, \& Sanders, 2016; Barengo et al., 2014; Faude, Rommers, \& Rössler, 2018; Thorborg et al., 2017).

Las lesiones en cadera e ingle representan el $14 \%$ de las lesiones totales en futbolistas profesionales, con una severidad muy variable (Ekstrand, Hägglund, \& Waldén, 2011b). El dolor inguinal en fútbol representa entre el 10-21\% de las lesiones totales (Ekstrand \& Hilding, 1999; Hägglund et al., 2005; Panasiuk, 2009; Salces et al., 2014; Waldén et al., 2005). Por otro lado, la UEFA, en fútbol profesional, cifra la incidencia de dolor inguinal en zona púbica y cadera en 1.1/1000h.: 3.5/1.000h en competición y 0.6/1.000h en entrenamiento (Walde \& Ekstrand, 2009), sin embargo, en jugadores sub-elite en fútbol (no profesional) representa $0.40 \mathrm{l} / 1000 \mathrm{~h}$ (Holmich, Thorborg, Nyvold, et al., 2014). Por lo tanto, se observa una gran diferencia por nivel, no profesional y profesional ( 0.40 a 1.1), aunque con ciertas discrepancias (Hägglund, Waldén, \& Ekstrand, 2016), y entre el entrenamiento y la competición (0.6 a 3.5). En cuanto a la demarcación, los mediocentros son los más afectados con un $42.8 \%$, mientras que los defensores y delanteros muestran incidencia similar con un 25.7\% (Rodriguez et al., 2001). La lesión supone 29.2 días promedio de baja competitiva (Noya \& Sillero, 2012b). En el estudio de Mosler et al. (2018) se refiere una tasa general de recurrencia del 20\%, 27\% para las lesiones de aparición repentina y del $18 \%$ para las lesiones de aparición gradual.

El dolor inguinal es llamado comúnmente pubalgía, termino erróneo debido a que aúna patologías afines como osteopatía dinámica de pubis u osteítis de pubis, hernia inguinal, hernia del deportista y tendinopatía de aductores que, además, en ocasiones no dan dolor únicamente en la zona del pubis. El dolor inguinal es un concepto más global que determina una consecuencia, no un diagnóstico, que puede generarse por decenas de causas con gran cantidad de diagnósticos según la literatura científica (Bisciotti et al., 2015; Elattar, Choi, Dills, \& Busconi, 2016; Robertson, Barker, Fahrer, \& Schache, 2009; Rodriguez, Miguel, Lima, \& Heinrichs, 2001). En este estudio, se considera más interesante hablar de dolor inguinal referido por el ejercicio, al igual que las últimas revisiones y estudios científicos más relevantes acerca de esta patología, aclarando así el objeto de estudio (Bisciotti et al., 2015; Branci et al., 2014; Elattar et al., 2016; Ryan, DeBurca, \& Mc Creesh, 2014; Schoberl et al., 2017). El dolor inguinal suele ser común en deportes que requieren un uso específico y excesivo de la musculatura flexora de la cadera, aunque la causa es difícil de determinar debido a la compleja anato- 
mía local y al amplio diagnóstico diferencial (Elattar et al., 2016).

La etiología de la lesión no es clara. Una de las hipótesis que se plantea es que la actividad no es coordinada, evitándose inhibiciones, una adecuada pre-activación y coactivación muscular, y un adecuado nivel de fuerza, reclutamiento y sincronización. Todo esto puede producir una serie de desajustes que pueden ser determinantes en la producción de esta patología (Cohen, Kleinhenz, Schiller, \& Tabaddor, 2016; Meyers, Yoo, \& Horner, 2008).

Según Brunker y Khan (2006) el dolor inguinal inespecífico relacionado con el ejercicio se ha diferenciado a muchos niveles; pero las definiciones más usadas y populares son osteítis del pubis y pubalgía atlética. La osteítis del pubis ha sido un término utilizado para cubrir el dolor inguinal relacionado con el ejercicio en atletas. Sin embargo, el dolor de larga evolución es raramente inflamatorio en su naturaleza, y los hallazgos de incremento de absorción en el hueso no es universal, por lo cual el termino osteítis del pubis parece inapropiado. Aunque este sigue siendo utilizado coloquialmente con frecuencia, en la literatura debería evitarse. Por lo tanto, para esta revisión, lo denominaremos "dolor inguinal relacionado con el ejercicio" (Verrall, Slavotinek, Fon, \& Barnes, 2007; Weir et al., 2015).

El dolor inguinal (“Groin Pain” GP) es una patología muy relacionada con la práctica deportiva y el fútbol. Es muy importante especificar que "dolor inguinal" significa "dolor en el área de la ingle". De acuerdo con las diferentes lesiones y los diferentes síntomas referidos por el paciente, podemos identificar diferentes tipos de dolor inguinal, con variedad de causas diferentes (Bisciotti et al., 2015). Los mecanismos desencadenantes de dolor inguinal son, por un lado, la falta de estabilización dinámica donde suele observarse afectación del tendón del aductor largo (44-66\%), lesión aislada de los rectos abdominales (27\%) y lesión completa de la aponeurosis conjunta (15-30\%) y, por otro, traumatismo directo (Naranjo, Bayo, Fernández \& Salas, 2012).

Los factores de riesgo principales de dolor inguinal, a nivel extrínseco, se basarían en el terreno de juego, demarcación y calzado (Leventer et al., 2016; Thomson et al., 2015; Williams et al., 2013), así como en la planificación y control del entrenamiento (Barrett et al., 2016; Ehrmann et al., 2016; Malone et al., 2018).

Por otro lado, las factores de riesgo intrínsecos estarían asociados con el rango de movilidad articular y la fuerza en cadera (Kloskowska et al., 2016; Mosler et al., 2015; Nevin \& Delahunt, 2014), lesiones previas
(Langhout et al., 2018; McCall et al., 2015) y el sexo (Orchard, 2015).

El sobreentrenamiento y el incorrecto acondicionamiento físico son situaciones desencadenantes de la patología, sobre todo en deportes de alto riesgo de este tipo de lesión, como en el caso del fútbol (Bastos et al., 2013; Bowen, Gross, Gimpel, \& Li, 2016; Casáis Martínez, 2008). Por todo ello, el entrenamiento debe de estar correctamente estructurado, bajo unos principios y organización de cargas y contenidos adecuados. La planificación de este trabajo de prevención debe ser grupal e individualizado, eliminando al máximo posibles factores de riesgo que aumentan la posibilidad de padecer esta lesión.

En el fútbol, la complejidad lesional determina la interrelación y retroalimentación de un sistema complejo en cuanto a nivel de fuerza, sinergias musculares, función, coordinación, coactivación y calidad de movimiento. Estos elementos deben trabajarse adecuadamente provocando un gesto deportivo de calidad, a partir de compensaciones estáticas y dinámicas acordes a las situaciones dadas en el juego (Bittencourt, Meeuwisse, Mendonça, Ocarino, \& Fonseca, 2016; Chena, Rodríguez, \& Cerezal, 2017; Quatman, Quatman, \& Hewet, 2009).

\section{Hipótesis y objetivos}

Se plantean dos hipótesis diferentes, la primera es que el déficit de fuerza y desequilibrio muscular son factores de alto riesgo para la producción y recidivas de lesiones de dolor inguinal en fútbol. Y la segunda es que las intervenciones mediante ejercicio son efectivas para la recuperación y prevención de dolor inguinal en futbolistas. El objetivo de este estudio fue identificar los factores de riesgo asociados a la producción y recidivas de dolor inguinal en fútbol y revisar la eficacia del tratamiento mediante ejercicio y las estrategias más efectivas para la prevención, rehabilitación, readaptación y vuelta a la práctica deportiva del futbolista.

\section{Método}

\section{Estrategia de búsqueda bibliográfica}

En este estudio se ha llevado a cabo una revisión bibliográfica de la literaura científica relacionada con el dolor inguinal. Para dicho proceso se utilizó la estrategia de búsqueda a través de los términos MeSH y términos libres más utilizados en la literatura. La estrategia de búsqueda realizada en PubMed se muestra a continuación: 
\#1 Osteitis pubis

\#2 Groin Pain

\#3 Soccer Injuries

\#4 Exercise Therapy [MeSH]

\#5 Return to Sport [MeSH]

\#6 \#1 OR \#2 OR \#3

\#7 \#4 OR \#5

\#8 \#6 AND \#7

Los estudios se filtraron por tipo de estudios observacionales, cohortes prospectivas, ensayos clínicos, revisiones sistemáticas y meta-análisis, con una antigüedad máxima de 5 años. Los estudios fueron elegidos a través de la lectura de título y resumen y siguiendo los criterios de selección.

\section{Criterios de inclusión y exclusión}

Los criterios de selección de los estudios de esta revisión fueron los siguientes:

- Criterios de inclusión:

1) Estudios observacionales o revisiones sistemáticas que identificaran los factores de riesgo de padecer dolor inguinal en futbolistas o estudios sobre intervenciones mediante ejercicio para la rehabilitación, prevención, readaptación y vuelta a la práctica deportiva de futbolistas.

2) Se incluirán estudios que utilicen otros tipos de intervención como complemento del tratamiento con ejercicio.

3) Estudios con población de futbolistas amateur o profesionales mayores de 18 años.

- Criterios de exclusión:

1) Los estudios de intervención, que no incluyan el tratamiento mediante ejercicio, para la rehabilitación, prevención o readaptación del dolor inguinal.

2) Poblaciones de no futbolistas.

\section{Resultados}

\section{Selección de estudios}

Tras la búsqueda y cribado de los estudios se identificaron para su selección un total de 120 estudios, de los cuales se incluyeron en esta revisión después de la lectura a texto completo un total de 31 (Fig. 1).

Factores de riesgo relacionados con el dolor inguinal

Las características de los estudios incluidos que trataron los factores de riesgo de dolor inguinal en futbolistas se muestran en la Tabla 1. El resumen de los resultados de los estudios incluidos se muestra en la Tabla 2.

Los resultados de esta revisión, en relación con los factores de riesgo, muestran que la falta de fuerza en la musculatura aductora de cadera, la distancia recorrida y el haber sufrido una lesión previa son los factores de riesgo más identificados en la literatura para el desarrollo de lesiones y producción de dolor inguinal en futbolistas. Sin embargo, la falta de ROM, el desequilibrio muscular y la estabilización lumbo-pélvica no se han podido determinar como factores de riesgo en base a la literatura incluida en esta revisión. Se han observado otros factores que pueden influir en el desarrollo de lesiones como el tipo césped, el calzado y el género.

Estudios destinados a la prevención de lesiones en deportistas

Las características de los estudios incluidos que trataron la prevención de lesiones en futbolistas se muestran en la Tabla 3.

En relación con las estrategias de prevención de lesiones, se ha observado que la realización de proto-

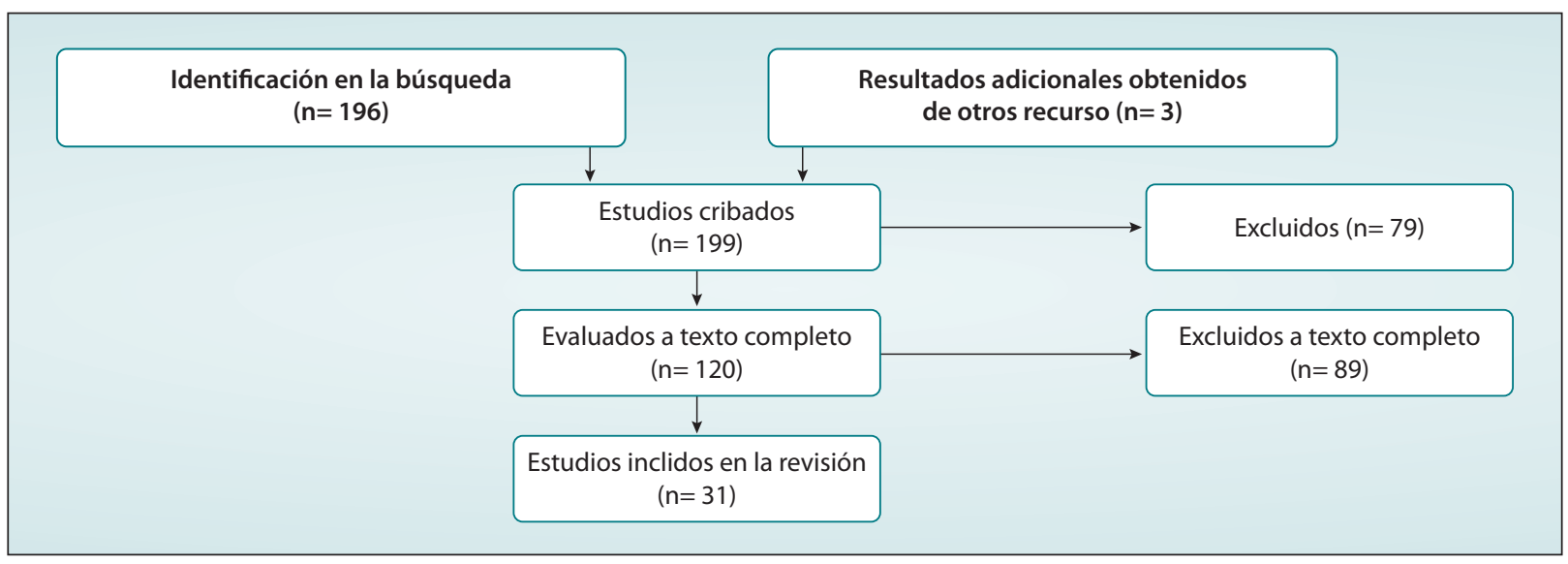

Figura 1. Diagrama de flujo, evaluación e inclusión-exclusión de estudios 
Tabla 1. Características de los estudios encontrados en referencia a los factores de riesgo de dolor inguinal en futbolistas

\begin{tabular}{|c|c|c|c|}
\hline Autor & Tipo & $\mathbf{N}$ & Resultados \\
\hline $\begin{array}{l}\text { Williams, Akogyrem, \& } \\
\text { Williams, } 2013\end{array}$ & Meta-análisis & $\begin{array}{l}25 \text { estudios } \\
\text { incluidos }\end{array}$ & $\begin{array}{l}20.26 \text { lesiones por } 1000 \text { horas en césped artificial. } 24.45 \text { lesiones por } 1000 \text { horas } \\
\text { en césped natural. } \\
\text { Césped Artificial VS Natural: (RR }=0.86(0.74-0.93, p<0.05)\end{array}$ \\
\hline Nevin \& Delahunt, 2014 & Casos-controles & $\begin{array}{l}16 \text { deportistas con } \\
\text { dolor inguinal de } \\
\text { larga evolución y } \\
16 \text { sujetos sanos }\end{array}$ & $\begin{array}{l}\text { Disminución de la rotación interna y externa de cadera en ambas piernas en los } \\
\text { jugadores con dolor inguinal en comparación a sujetos sanos. } \\
\text { Disminución de fuerza medida con el squeeze test para los aductores en los } \\
\text { jugadores con dolor inguinal en comparación a sujetos sanos. }\end{array}$ \\
\hline $\begin{array}{l}\text { Mosler, Agricola, Weir, } \\
\text { Holmich, \& Crossley, } 2015\end{array}$ & $\begin{array}{l}\text { Revisión sistemática } \\
\text { y meta-análisis }\end{array}$ & $\begin{array}{l}17 \text { estudios } \\
\text { incluidos }\end{array}$ & $\begin{array}{l}\text { Diferencias en el dolor al realizar el squeeze test, single aductor test, el test } \\
\text { bilateral de aductores, la elevación de la pierna activa e impingment test en } \\
\text { comparación de atletas con dolor inguinal y sujetos sanos. } \\
\text { Disminución de la rotación interna de cadera en comparación con sujetos sanos. } \\
\text { No se encontraron diferencias significativas en la disminución de la rotación } \\
\text { externa de cadera } \\
\text { No diferencias en la fuerza abductora entre sujetos sanos y con dolor. } \\
\text { Diferencias en la fuerza de flexión y ratio de flexo/extensores entre sujetos } \\
\text { sanos y con dolor. } \\
\text { Las diferencias en la función del tronco (fuerza y control) no parece ser } \\
\text { concluyente }\end{array}$ \\
\hline Orchard, 2015 & Revisión sistemática & $\begin{array}{l}31 \text { estudios } \\
\text { incluidos }\end{array}$ & $\begin{array}{l}\text { Mayor riesgo relativo en hombres que en mujeres de sufrir una lesión o dolor } \\
\text { inguinal. }\end{array}$ \\
\hline $\begin{array}{l}\text { Thomson, Whiteley, \& } \\
\text { Bleakley, } 2015\end{array}$ & $\begin{array}{l}\text { Revisión sistemática } \\
\text { y meta-análisis }\end{array}$ & $\begin{array}{l}4972 \text { atletas } \\
\text { incluidos }\end{array}$ & $\begin{array}{l}\text { Una mayor tracción rotacional está asociado a un riesgo } 2.5 \text { veces mayor de } \\
\text { lesión en la extremidad inferior. }\end{array}$ \\
\hline McCall et al., 2015 & Revisión sistemática & $\begin{array}{l}14 \text { estudios } \\
\text { incluidos }\end{array}$ & $\begin{array}{l}\text { Encontraron como factores de riesgo el haber sufrido una lesión previa y la } \\
\text { fatiga por opinión de expertos. } \\
\text { El desequilibrio muscular es inconcluyente }\end{array}$ \\
\hline $\begin{array}{l}\text { Leventer, Eek, Hofstetter, \& } \\
\text { Lames, } 2016\end{array}$ & $\begin{array}{l}\text { Estudio retrospectivo } \\
\text { observacional }\end{array}$ & $\begin{array}{l}1448 \text { jugadores de } \\
\text { fútbol masculinos }\end{array}$ & $\begin{array}{l}\text { Mayor incidencia de lesiones para los centrales (defensas) y centro campistas } \\
\text { (extremo). } \\
\text { La incidencia de lesión inguinal es menor en defensas (laterales/extremo) y } \\
\text { porteros que en los delanteros. } \\
\text { Las lesiones inguinales son las que producen más pérdidas de días en los } \\
\text { delanteros. }\end{array}$ \\
\hline Barrett et al., 2016 & $\begin{array}{l}\text { Estudio de cohorte } \\
\text { observacional }\end{array}$ & $\begin{array}{l}574 \text { observaciones } \\
\text { de partido }\end{array}$ & $\begin{array}{l}\text { La eficiencia motora es menor durante las últimas etapas de cada mitad de un } \\
\text { partido, observándose un aumento de la incidencia de lesiones y fatiga. }\end{array}$ \\
\hline $\begin{array}{l}\text { Ehrmann, Duncan, } \\
\text { Sindhusake, Franzsen, \& } \\
\text { Greene, } 2016\end{array}$ & $\begin{array}{l}\text { Estudio } \\
\text { observacional }\end{array}$ & $\begin{array}{l}19 \text { jugadores de } \\
\text { elite de fútbol }\end{array}$ & $\begin{array}{l}\text { Previamente a la lesión se ha observado que en la última semana hay un } \\
\text { aumento } 9.6 \% \text { de los metros por minuto recorridos en los jugadores } \\
\text { En un periodo de cuatro semanas un aumento del } 7.4 \% \text { de los metros por } \\
\text { minutos precede a la lesión. } \\
\text { Una disminución de la carga ( } 15.4 \text { y } 9 \% \text { para } 1 \text { y } 4 \text { semanas) es un precedente } \\
\text { de lesiones. La justificación de este hecho se debe a la falta de preparación. }\end{array}$ \\
\hline $\begin{array}{l}\text { Kloskowska, Morrissey, } \\
\text { Small, Malliaras, \& Barton, } \\
2016\end{array}$ & Revisión sistemática & $\begin{array}{l}17 \text { estudios } \\
\text { observacionales } \\
\text { (8 retrospectivos, } \\
9 \text { prospectivos). }\end{array}$ & $\begin{array}{l}\text { Resultados estudios prospectivos: } \\
\text { - Disminución de la abducción de cadera } \\
\text { - La rotación total de ambas caderas es un factor de riesgo para el desarrollo } \\
\text { del dolor inguinal. } \\
\text { - Disminución de la fuerza aductora medida con isocinético. } \\
\text { - Una diminución de la fuerza de flexión de la rodilla isocinética es un factor } \\
\text { de riesgo, a una velocidad de } 60 \% / 5 \text {. } \\
\text { - Disminución de la fuerza abductora en velocidad angular en } 30 \% \text { s pero no en } \\
119 \% \text { y } 210^{\circ} / s \\
\text { - No se encontraron relaciones entre el rango de movimiento de la cadera en } \\
\text { rotación interna o externa, ni la fuerza de extensión de la rodilla medida con } \\
\text { isocinético. } \\
\text { Resultados estudios retrospectivos: } \\
\text { - Disminución de la fuerza de aducción de cadera a } 45^{\circ} \text { con squeeze test. } \\
\text { - No relación entre la fuerza de la musculatura abductora de cadera } \\
\text { - Limitada evidencia sobre si la disminución del grosor del transverso del } \\
\text { abdomen, ratio aductor/abductor estén asociado al dolor inguinal. }\end{array}$ \\
\hline Tak et al., 2017 & Revisión sistemática & $\begin{array}{l}7 \text { estudios } \\
\text { prospectivos y } 4 \\
\text { casos controles }\end{array}$ & $\begin{array}{l}\text { La rotación total de ambas caderas por debajo de } 85^{\circ} \text { es un factor de riesgo } \\
\text { para el desarrollo del dolor inguinal. } \\
\text { La rotación interna, la abducción y extensión de cadera no están asociadas con } \\
\text { el riesgo o presencia de dolor inguinal. }\end{array}$ \\
\hline $\begin{array}{l}\text { Malone, Owen, Newton, } \\
\text { Mendes, Collins \& Gabbett, } \\
2017\end{array}$ & $\begin{array}{l}\text { Estudio de cohorte } \\
\text { observacional }\end{array}$ & $\begin{array}{l}48 \text { jugadores } \\
\text { profesionales de } \\
\text { fútbol }\end{array}$ & $\begin{array}{l}\text { Relación trabajo agudo: crónica de entre } 1.00 \text { y } 1.25 \text { determina menor riesgo de } \\
\text { lesión. Relación a tener en cuenta también en pretemporada, cargas y relación } \\
\text { carga aguda:crónica moderadas. Una mayor capacidad aeróbica intermitente } \\
\text { previene de lesiones frente a cambios rápidos en la carga de trabajo en } \\
\text { jugadores de fútbol de élite. }\end{array}$ \\
\hline Malone et al., 2018 & $\begin{array}{l}\text { Estudio de cohorte } \\
\text { observacional }\end{array}$ & $\begin{array}{l}37 \text { jugadores de } \\
\text { elite de fútbol }\end{array}$ & $\begin{array}{l}\text { Exponer a los jugadores a grandes y rápidos incrementos de la distancia de } \\
\text { sprint de alta velocidad y carrera en sprint es un factor de riesgo }\end{array}$ \\
\hline Langhout et al., 2018 & $\begin{array}{l}\text { Estudio de cohorte } \\
\text { observacional } \\
\text { prospectivo y } \\
\text { retrospectivo }\end{array}$ & $\begin{array}{l}190 \text { jugadores } \\
\text { profesionales de } \\
\text { fútbol }\end{array}$ & $\begin{array}{l}\text { Haber padecido una lesión (cualquier lesión) es un factor de riesgo para } \\
\text { desarrollar dolor inguinal. } \\
\text { El rango de movilidad no se identificó como un factor de riesgo para el } \\
\text { desarrollo de dolor inguinal. }\end{array}$ \\
\hline
\end{tabular}


Tabla 2. Resumen de los principales factores de riesgo de dolor inguinal en futbolistas

\begin{tabular}{|c|c|}
\hline Factor & Probabilidad/Rango de lesión \\
\hline Tipo de césped & $\begin{array}{l}\text { Menor probabilidad de lesión en césped artificial que en césped natural } \\
(\mathrm{RR}=0.86(0.74-0.93, p<0.05) \text { (Williams et al., 2013). }\end{array}$ \\
\hline Posición de juego & $\begin{array}{l}\text { Mayor incidencia de lesiones inguinales y número de días perdidos en delanteros frente a otras } \\
\text { posiciones (Leventer et al., 2016). }\end{array}$ \\
\hline Calzado deportivo & $\begin{array}{l}\text { Mayor tracción rotacional (Thomson et al., 2015) produce una mayor incidencia de lesiones en el } \\
\text { miembro inferior. }\end{array}$ \\
\hline Género & Mayor riesgo en hombre que en mujeres (RR: 2.45) (Orchard, 2015). \\
\hline $\begin{array}{l}\text { Movilidad en rotación interna } \\
\text { de cadera }\end{array}$ & $\begin{array}{l}\text { Disminución de rotación interna de cadera (Mosler et al., 2015; Nevin \& Delahunt, 2014). } \\
\text { No se encontró asociación ente la rotación interna y dolor inguinal (Kloskowska et al., 2016; Langhout } \\
\text { et al., 2018; Tak et al., 2017). } \\
\text { Disminución de la rotación total }\left(<85^{\circ}\right) \text { de ambas caderas es un factor de riesgo para el desarrollo de } \\
\text { dolor inguinal (Kloskowska et al., 2016; Tak et al., 2017). }\end{array}$ \\
\hline $\begin{array}{l}\text { Movilidad en rotación externa } \\
\text { de cadera }\end{array}$ & $\begin{array}{l}\text { Disminución de la rotación externa en comparación a sujetos sanos (Nevin \& Delahunt, 2014). } \\
\text { No encontró diferencias en la rotación externa en comparación a sujetos sanos (Mosler et al., 2015). } \\
\text { No se encontró asociación ente la rotación externa y dolor inguinal (Kloskowska et al., 2016; Langhout } \\
\text { et al., 2018). }\end{array}$ \\
\hline $\begin{array}{l}\text { Movilidad en abducción de } \\
\text { cadera }\end{array}$ & $\begin{array}{l}\text { Disminución de la abducción de cadera es un posible factor de riesgo (Kloskowska et al., 2016). } \\
\text { Disminución de la abducción de cadera no tiene asociación con el desarrollo de dolor inguinal (Tak et } \\
\text { al., 2017). }\end{array}$ \\
\hline Fuerza de aductores de cadera & $\begin{array}{l}\text { Disminución de fuerza y dolor con Squeeze Test en comparación a sujetos sanos (Mosler et al., 2015; } \\
\text { Nevin \& Delahunt, 2014). } \\
\text { Disminución de la fuerza con Squeeze Test e isocinético (Kloskowska et al., 2016). }\end{array}$ \\
\hline Fuerza de abductores de cadera & Inconcluyente (Kloskowska et al., 2016). \\
\hline Fuerza de extensión de rodilla & No se encontró relación (Kloskowska et al., 2016). \\
\hline Fuerza de flexión de rodilla & Se encontró relación (Kloskowska et al., 2016). \\
\hline Distancia recorrida & $\begin{array}{l}\text { Previamente a la lesión se ha observado que en la última semana hay un aumento }(9.6 \%) \text { de los } \\
\text { metros por minuto recorridos en los jugadores (Ehrmann et al., 2016). } \\
\text { Cambios en aumento en la distancia recorrida de alta velocidad de sprint (351-455m) y carrera de } \\
\text { sprint }(75-105 \mathrm{~m}) \text { es un factor de riesgo de lesión (Malone et al., 2018). }\end{array}$ \\
\hline Carga & $\begin{array}{l}\text { Una disminución de la carga media de una temporada (15.4 y } 9 \% \text { para } 1 \text { y } 4 \text { semanas) es un precedente } \\
\text { de lesiones. La justificación de este hecho se debe a la falta de preparación (Ehrmann et al., 2016). }\end{array}$ \\
\hline Fatiga & $\begin{array}{l}\text { La fatiga durante el partido influye en la lesiones (opinión de expertos) (McCall et al., 2015). } \\
\text { La eficiencia locomotora en las últimas etapas de las partes de cada tiempo es menor, lo cual es un } \\
\text { marcador de fatiga y relacionable con una mayor incidencia de lesiones en estos periodos (Barrett et } \\
\text { al., 2016). }\end{array}$ \\
\hline Lesión previa & $\begin{array}{l}\text { Haber sufrido una lesión previa se identificó como factor de riesgo (Langhout et al., 2018; McCall et } \\
\text { al., 2015). }\end{array}$ \\
\hline Desequilibrio muscular & No concluyente (Kloskowska et al., 2016; McCall et al., 2015). \\
\hline CORE & No concluyente (Kloskowska et al., 2016; McCall et al., 2015). \\
\hline
\end{tabular}

colos generales destinados a la mejora de la fuerza y control neuromuscular disminuye la incidencia de lesiones y mejora otras cualidades fisiológicas. El protocolo de FIFA 11 + parece ser el protocolo de prevención de lesiones más estudiado y eficaz para la disminución de lesiones en deportistas (disminución entre un 12 y $70 \%$ de las lesiones en general).

Estudios destinados a la recuperación de lesiones en deportistas

Las características de los estudios incluidos que trataron la recuperación de lesiones en futbolistas se muestran en la Tabla 4.
En cuanto a las intervenciones destinadas a la recuperación de las lesiones inguinales se ha observado que el ejercicio terapéutico parece ser una herramienta eficaz para la recuperación y tratamiento de los deportistas con este dolor. La combinación del ejercicio terapéutico con otras modalidades de tratamiento físico (terapia manual u ondas de choque) parecen mejorar la efectividad de los protocolos y recuperación de los deportistas.

\section{Discusión}

Tras identificar los factores de riesgo asociados a la producción y recidivas de dolor inguinal en fútbol y 
Tabla 3. Características de los estudios sobre prevención de lesiones en futbolistas

\begin{tabular}{|c|c|c|c|c|}
\hline Autor & Tipo de estudio & $\mathbf{N}$ & Intervención & Resultados \\
\hline $\begin{array}{l}\text { (Owen et al., } \\
\text { 2013) }\end{array}$ & $\begin{array}{l}\text { Estudio } \\
\text { experimental }\end{array}$ & $\begin{array}{l}26 \text { y } 23 \text { jugadores } \\
\text { de élite de fútbol } \\
\text { de la liga escocesa }\end{array}$ & $\begin{array}{l}\text { Programa de ejercicios de } \\
\text { equilibrio, fuerza funcional y } \\
\text { estabilidad lumbo-pélvica }\end{array}$ & $\begin{array}{l}\text { Reducción de un } 43 \% \text { de lesiones cuando } \\
\text { en comparación con el control }\end{array}$ \\
\hline $\begin{array}{l}\text { (Bizzini et al., } \\
\text { 2013) }\end{array}$ & $\begin{array}{l}\text { Estudio } \\
\text { experimental }\end{array}$ & $\begin{array}{l}20 \text { jugadores } \\
\text { masculinos amateur } \\
\text { de fútbol }\end{array}$ & FIFA $11+$ & $\begin{array}{l}\text { Diferencias en lactato, VO2, temperatura } \\
\text { del core, ratio de fuerza, star excursión } \\
\text { balance test y countermovement jump. }\end{array}$ \\
\hline $\begin{array}{l}\text { (Impellizzeri et } \\
\text { al., 2013) }\end{array}$ & $\begin{array}{l}\text { Estudio } \\
\text { experimental }\end{array}$ & $\begin{array}{l}81 \text { jugadores en dos } \\
\text { grupos }\end{array}$ & $\begin{array}{l}\text { FIFA } 11+(n=42) \\
\text { Grupo control }(n=39)\end{array}$ & $\begin{array}{l}\text { Diferencias significativas para el grupo FIFA } \\
11+\text { después de } 9 \text { semanas de entrenamien- } \\
\text { to, en sprint y estabilidad del CORE. }\end{array}$ \\
\hline $\begin{array}{l}\text { (Mayo, Seijas, \& } \\
\text { Álvarez, 2014) }\end{array}$ & $\begin{array}{l}\text { Revisión } \\
\text { sistemática y } \\
\text { meta-análisis }\end{array}$ & 6 estudios & FIFA $11+$ & $\begin{array}{l}\text { Reducción en un } 12 \% \text { de las lesiones en } \\
\text { la región inguinal en deportistas de élite } \\
\text { jóvenes. }\end{array}$ \\
\hline $\begin{array}{l}\text { (Barengo et al., } \\
\text { 2014) }\end{array}$ & $\begin{array}{l}\text { Revisión } \\
\text { sistemática }\end{array}$ & 12 estudios & FIFA $11+$ & $\begin{array}{l}\text { Reducción de lesiones en un rango de } \\
30-70 \%\end{array}$ \\
\hline $\begin{array}{l}\text { (Silvers-Granelli } \\
\text { et al., 2015) }\end{array}$ & $\begin{array}{l}\text { Ensayo clínico } \\
\text { aleatorizado }\end{array}$ & $\begin{array}{l}850 \text { jugadores en } \\
\text { el grupo control y } \\
675 \text { jugadores en el } \\
\text { grupo experimental }\end{array}$ & $\begin{array}{l}\text { FIFA } 11+(n=675) \\
\text { Grupo control }(n=850)\end{array}$ & $\begin{array}{l}\text { Reducción de un } 46.1 \% \text { y una disminución } \\
\text { del tiempo perdido debido a la lesión un } \\
28.6 \%\end{array}$ \\
\hline $\begin{array}{l}\text { (Hammes et al., } \\
\text { 2015) }\end{array}$ & $\begin{array}{l}\text { Ensayo } \\
\text { aleatorizado }\end{array}$ & $\begin{array}{l}\text { Jugadores } \\
\text { veteranos mayores } \\
\text { de } 35 \text { años }(n=383)\end{array}$ & $\begin{array}{l}\text { FIFA } 11+(n=146) \\
\text { Grupo control }(n=119)\end{array}$ & $\begin{array}{l}\text { No diferencias en la ratio de lesiones } \\
\text { general, pero si en la incidencia de } \\
\text { lesiones severas. }\end{array}$ \\
\hline $\begin{array}{l}\text { (Esteve, } \\
\text { Rathleff, Bagur- } \\
\text { Calafat, Urrutia, } \\
\text { \& Thorborg, } \\
\text { 2015) }\end{array}$ & $\begin{array}{l}\text { Revisión } \\
\text { sistemática y } \\
\text { meta-análisis }\end{array}$ & $\begin{array}{l}7 \text { ensayos clínicos }(6 \\
\text { en fútbol) } \\
(n=4191)\end{array}$ & $\begin{array}{l}\text { Diferentes tipos de } \\
\text { intervención, programas de } \\
\text { fuerza y estabilización }\end{array}$ & $\begin{array}{l}\text { No se encontraron diferencias } \\
\text { significativas en el RR }(0.48-0.81) \text { de sufrir } \\
\text { una lesión inguinal. } \\
\text { Disminución del } 19 \% \text { de lesiones } \\
\text { inguinales (clínicamente relevante) }\end{array}$ \\
\hline $\begin{array}{l}\text { (Al Attar, } \\
\text { Soomro, Pappas, } \\
\text { Sinclair, \& } \\
\text { Sanders, 2016) }\end{array}$ & $\begin{array}{l}\text { Revisión } \\
\text { sistemática y } \\
\text { meta-análisis }\end{array}$ & - & Programa FIFA / FIFA 11+ & $\begin{array}{l}\text { Reducción del riesgo de sufrir una lesión y } \\
\text { de sufrir una lesión en el miembro inferior. } \\
\text { El Programa FIFA } 11+\text { mantiene una } \\
\text { disminución del } 30 \text { a } 50 \% \text { de disminución } \\
\text { de lesiones en seguimientos a largo plazo } \\
\text { comparado con los FIFA. }\end{array}$ \\
\hline $\begin{array}{l}\text { (Charlton, Drew, } \\
\text { Mentiplay, } \\
\text { Grimaldi, \& } \\
\text { Clark, 2017) }\end{array}$ & $\begin{array}{l}\text { Revisión } \\
\text { sistemática }\end{array}$ & $\begin{array}{l}14 \text { estudios } \\
\text { incluidos }\end{array}$ & Ejercicio terapéutico & $\begin{array}{l}\text { Limitada evidencia (nivel } 2-3 \text { ) que el } \\
\text { ejercicio terapéutico pueda reducir la } \\
\text { incidencia de lesiones inguinales. }\end{array}$ \\
\hline $\begin{array}{l}\text { (Gomes Neto et } \\
\text { al., 2017) }\end{array}$ & $\begin{array}{l}\text { Revisión } \\
\text { sistemática y } \\
\text { meta-análisis }\end{array}$ & $\begin{array}{l}11 \text { estudios } \\
\text { incluidos ( } 4700 \\
\text { participantes) }\end{array}$ & FIFA 11+ & $\begin{array}{l}\text { Reducción en el riesgo de lesión. Mejoría } \\
\text { en el equilibrio dinámico. Mejora en la } \\
\text { agilidad. No mejoría en la altura de salto. } \\
\text { No mejoría en el sprint }\end{array}$ \\
\hline $\begin{array}{l}\text { (Thorborg et al., } \\
\text { 2017) }\end{array}$ & $\begin{array}{l}\text { Revisión } \\
\text { sistemática y } \\
\text { meta-análisis }\end{array}$ & $\begin{array}{l}6 \text { ensayos } \\
\text { aleatorizados }\end{array}$ & $\begin{array}{l}2 \text { FIFA } 11 \\
4 \text { FIFA } 11+\end{array}$ & $\begin{array}{l}\text { Reducción del riesgo de lesión en general } \\
\text { (disminución de un } 39 \% \text { ) }\end{array}$ \\
\hline $\begin{array}{l}\text { (Sadigursky et } \\
\text { al., 2017) }\end{array}$ & $\begin{array}{l}\text { Revisión } \\
\text { sistemática y } \\
\text { meta-análisis }\end{array}$ & $\begin{array}{l}6344 \text { jugadores de } \\
\text { fútbol }\end{array}$ & FIFA 11+ & $\begin{array}{l}\text { Reducción del riesgo de lesión en general } \\
\text { (disminución de un } 30 \% \text { ) }\end{array}$ \\
\hline
\end{tabular}

revisar la eficacia del tratamiento mediante ejercicio y las estrategias más efectivas para la prevención, rehabilitación, readaptación y vuelta a la práctica deportiva del futbolista, como objetivo de este estudio, se discuten estos resultados en tres apartados:

\section{Factores de riesgo}

Los factores de riesgo más relevantes en el dolor inguinal en futbolistas pueden ser tanto intrínsecos como extrínsecos. Los hombres tienen un mayor ries- go relativo que las mujeres de sufrir dolor inguinal (Orchard, 2015), además la producción de cualquier lesión previa es un factor de riesgo de dolor inguinal (Langhout et al., 2018; McCall et al., 2015).

En competición, la fatiga producida, sobre todo, en las fases finales de cada parte del partido es un factor de riesgo (McCall et al., 2015; Barrett et al., 2016) y en entrenamiento las modificaciones sustanciales de la carga semanal pueden suponer un factor de riesgo desencadenante de la lesión. Estos pueden ser cambios en la carga externa o física, es decir, la distancia máxima 
Tabla 4. Características de los estudios sobre recuperación de lesiones en futbolistas

\begin{tabular}{|c|c|c|c|}
\hline Autor & Tipo de estudio & $\mathbf{N}$ & Resultados \\
\hline $\begin{array}{l}\text { (Almeida, Silva, } \\
\text { Andriolo, Atallah, \& } \\
\text { Peccin, 2013) }\end{array}$ & $\begin{array}{l}\text { Revisión } \\
\text { sistemática y } \\
\text { meta-análisis }\end{array}$ & 7 artículos incluidos & $\begin{array}{l}\text { Ejercicio terapéutico VS Fisioterapia convencional } \\
\text { A las } 16 \text { semanas para el dolor al descanso, palpación o durante la } \\
\text { actividad ( } R R=2.50 p=0.001) \text { a favor del ejercicio terapéutico } \\
\text { A las } 16 \text { semanas para la vuelta a la práctica deportiva ( } R R=5.95 \\
p=0.0002) \text { a favor del ejercicio terapéutico } \\
\text { Ejercicio terapéutico VS Terapia multi-modal } \\
\text { No diferencias entre la terapia multimodal y el ejercicio terapéutico } \\
\text { en el dolor al descanso, palpación o durante la actividad ( } R R=1.01 \text {, } \\
p=0.96 \text { ) } \\
\text { Para vuelta a la práctica deportiva ( } R R=0.92 p=0.75) \text { no se } \\
\text { encontraron diferencias significativas, pero si en el tiempo de la } \\
\text { vuelta a la práctica deportiva ( } 12.6 \text { semanas frente } 17.3 \text { semanas } \\
M D=-4.5 \text { semanas, } p=0.03 \text { ) a favor del grupo de terapia multimodal. }\end{array}$ \\
\hline $\begin{array}{l}\text { (King, Ward, Small, } \\
\text { Falvey, \& Franklyn- } \\
\text { Miller, 2015) }\end{array}$ & $\begin{array}{l}\text { Revisión } \\
\text { sistemática y } \\
\text { meta-análisis }\end{array}$ & 3332 atletas incluidos & $\begin{array}{l}\text { Rehabilitación VS Cirugía } \\
\text { La rehabilitación proporciona una recuperación más rápida que la } \\
\text { cirugía ( } 10.5 \text { semanas frente a } 23.1 \text { semanas) }\end{array}$ \\
\hline (Brown et al., 2016) & $\begin{array}{l}\text { Revisión } \\
\text { sistemática y } \\
\text { meta-análisis }\end{array}$ & $\begin{array}{l}1 \text { estudio de dolor } \\
\text { inguinal }\end{array}$ & $\begin{array}{l}\text { Ejercicio terapéutico VS Programa multimodal de fisioterapia } \\
\text { El grupo de ejercicio obtuvo mejores resultados a las } 16 \text { semanas. }\end{array}$ \\
\hline $\begin{array}{l}\text { (Cheatham, Kolber, \& } \\
\text { Shimamura, 2016) }\end{array}$ & $\begin{array}{l}\text { Revisión } \\
\text { sistemática }\end{array}$ & $\begin{array}{l}4 \text { estudios de series } \\
\text { de casos }\end{array}$ & $\begin{array}{l}\text { Programa de ejercicio más terapia manual } \\
\text { Encontraron que la combinación de programa de ejercicio } \\
\text { terapéutico basado en la mejora de la fuerza más terapia manual } \\
\text { (3-14 semanas) eran efectivos para la vuelta a la práctica deportiva } \\
\text { de los jugadores }\end{array}$ \\
\hline $\begin{array}{l}\text { (Charlton et al., } \\
\text { 2017) }\end{array}$ & $\begin{array}{l}\text { Revisión } \\
\text { sistemática }\end{array}$ & 14 estudios incluidos & $\begin{array}{l}\text { Fuerte evidencia (estudios nivel 4) que el ejercicio terapéutico como } \\
\text { un tratamiento para las lesiones inguinales en atletas en términos de } \\
\text { remisión de síntoma, vuelta a la práctica deportiva y recurrencias. }\end{array}$ \\
\hline $\begin{array}{l}\text { (Schoberl et al., } \\
\text { 2017) }\end{array}$ & $\begin{array}{l}\text { Ensayo clínico } \\
\text { aleatorizado a } \\
\text { doble ciego }\end{array}$ & $\begin{array}{l}44 \text { jugadores } \\
\text { amateurs de fútbol } \\
\text { con dolor inguinal }\end{array}$ & $\begin{array}{l}\text { Comparación de rehabilitación con VS sin ondas de choque. } \\
\text { Diferencias en el dolor en aquellos jugadores que recibieron las } \\
\text { ondas de choque frente a los que no al mes y } 3 \text { meses, no al año. } \\
\text { Diferencias en la discapacidad lumbar (Oswestry) al año del } \\
\text { tratamiento a favor del grupo con ondas de choque. } \\
\text { Diferencias en la discapacidad de cadera (HOOS) al mes, } 3 \text { meses y al } \\
\text { año del tratamiento. }\end{array}$ \\
\hline
\end{tabular}

recorrida, los metros por minuto o la longitud recorrida a alta intensidad, en la carga interna o fisiológica, por ejemplo, cambios en la frecuencia cardiaca, la temperatura corporal o el ritmo de sudoración o en la carga psicológica. Se ha demostrado cómo los metros promedio por minuto, cuando aumentan significativamente en comparación con el promedio de la temporada, en la relación carga aguda-crónica, la disminución significativa de la carga media de la temporada y el aumento en la distancia recorrida a velocidad de carrera de alta intensidad y carrera de sprint son predictores de lesiones de partes blandas (Ehrmann, Duncan, Sindhusake, Franzsen, \& Greene, 2016; Malone et al., 2018; Malone et al. 2017). En cuanto a la posición de juego, los delanteros son los jugadores con mayor incidencia de dolor inguinal en comparación con el resto de posiciones (Leventer, Eek, Hofstetter, \& Lames, 2016), posiblemente debido a la alternancia de repetición de acciones de alta intensidad y corta duración.

Por último, a nivel funcional, existen mayores discrepancias. La disminución de ROM, sobre todo en rotación interna, así como lo valores de fuerza en aductores obtenidos en deportistas lesionados (Nevin \& Delahunt, 2014; Mosler et al., 2015; Kloskowska, at al., 2016), al igual que la fuerza de flexión, desequilibrio ratio de flexo/extensores y el dolor muscular al activar zona inguinal ( Kloskowska et al., 2016; McCall et al., 2015; Mosler et al., 2015) y la abducción de cadera y flexión de rodilla (Kloskowska, at al., 2016) se observan como factores de riesgo principales. Aunque existen contradicciones con otros estudios que no determinan la rotación interna, abducción y extensión de cadera (Tak et al., 2017; Kloskowska et al., 2016), la fuerza de extensión de la rodilla (Kloskowska et al., 2016), ni la fuerza en la musculatura abductora, la función del tronco (Mosler et al., 2015) o el desequilibrio muscular (McCall et al., 2015) como factores de riesgo principales. También existe cierta discrepancia en cuanto a la limitación de valores de rotación externa con estudios que sí observan diferencia significativas entre sujetos lesionados y sanos (Nevin \& De- 
lahunt, 2014) y otros que no determinan la disminución de la rotación externa de cadera como un factor determinante (Mosler et al., 2015).

Otros estudios indican que la rotación total limitada de ambas caderas es un factor de riesgo (Tak et al., 2017; Kloskowska, at al., 2016), a diferencias de otras que indican que el rango de movilidad no es un factor de riesgo (Langhout et al., 2018; Kloskowska, at al., 2016).

Además, existe limitada evidencia sobre si la disminución del grosor del transverso del abdomen y ratio aductor/abductor está asociada al dolor inguinal (Kloskowska, at al., 2016).

\section{Prevención de lesiones}

Los programas de prevención de dolor inguinal referido al deporte más efectivos son los programas de ejercicio con contenidos de equilibrio, fuerza funcional y estabilidad lumbo-pélvica (Owen et al., 2013). Una estrategia común utilizada en la prevención de lesiones a nivel general son los protocolos de prevención, en este caso, el protocolo FIFA11+ es el más estudiado en la bibliografía, determinando unos beneficios en la prevención general y en la prevención especifica de dolor inguinal como pueden ser los valores y ratios de fuerza, las mejoras en la estabilidad y control postural (Bizzini et al., 2013; Impellizzeri et al., 2013), equilibrio dinámico y agilidad (Gomes Neto et al., 2017). Otros estudios no encuentran diferencias en valores de fuerza en altura de salto y sprint (Gomes Neto et al., 2017).

La reducción de la incidencia de lesiones con este tipo de estrategia posee un abanico muy extenso de entre 30-70\% (Al Attar, Soomro, Pappas, Sinclair, \& Sanders, 2016; Barengo et al., 2014; Gomes-Neto et al., 2017; Sadigursky et al., 2017; Silvers-Granelli et al., 2015; Thorborg et al., 2017), consiguiendo también una reducción del tiempo de recuperación debido a estas lesiones de un 28.6\% (Silvers-Granelli et al., 2015). En el dolor inguinal encontramos referencias directas de reducción de la lesión en un 12\% (Mayo, Seijas, \& Álvarez, 2014), por lo que puede ser una estrategia general complementaria y válida para la prevención de dolor inguinal, teniendo en cuenta la importancia de la individualización.

Sin embargo, también se encuentran discrepancias al evaluar diferentes tipos de intervención, programas de fuerza y estabilidad, donde no se encuentran diferencias significativas en la prevención de la incidencia de dolor inguinal (Esteve, Rathleff, Bagur-Calafat, Urrutia, \& Thorborg, 2015), aunque si se observa una reducción del 19\% de lesiones inguinales. El ejercicio terapéutico posee una evidencia limitada en la prevención del dolor inguinal (Charlton, Drew, Mentiplay, Grimaldi \& Clark, 2017).

Tratamiento, recuperación y readaptación físicodeportiva de dolor inguinal

El tratamiento basado en rehabilitación funcional proporciona una recuperación más rápida que la cirugía (King, Ward, Small, Falvey, \& Franklyn-Miller, 2015).

Los programas de ejercicio terapéutico y multimodales poseen mayores resultados, así como valores de umbral y percepción del dolor mejores, y valores de vuelta a la competición que el tratamiento convencional (Almeida, Silva, Andriolo, Atallah \& Peccin, 2013; Brown et al., 2016), sin embargo, el tratamiento multimodal favorece una recuperación más rápida que el ejercicio terapéutico aislado (Almeida, Silva, Andriolo, Atallah \& Peccin, 2013).

El tratamiento de fisioterapia convencional parece descartarse frente a los dos anteriores (Almeida, Silva, Andriolo, Atallah \& Peccin, 2013), ya que el trabajo combinado de ejercicio terapéutico y terapia manual se presenta como efectivo para la recuperación competitiva del deportista (Cheatham, Kolber, \& Shimamura, 2016). Además de ello, la inclusión de ejercicio terapéutico en la recuperación favorece la remisión de síntoma, vuelta a la práctica deportiva y recurrencias de la lesión (Charlton et al., 2017). Por último, las ondas de choque se observan adecuadas para la reducción del dolor a corto y medio plazo, en la mejora de la discapacidad lumbar (Oswestry) a largo plazo y en la discapacidad de cadera (HOOS) (Schoberl et al., 2017).

A nivel general, los contenidos utilizados en la intervención en prevención, rehabilitación o readaptación físico-deportiva deberán seguir una organización en fases y elementos adecuada (Schoberl et al., 2017). En caso de presencia de mucho dolor, el primer paso se centrará en el control de este a partir de tratamiento médico-fisioterapéutico con tratamiento farmacológico e intervenciones manuales, terapias físicas, técnicas invasivas (punción seca o EPI) y uso de otras técnicas complementarias (Topol, Reeves y Hassanein, 2005; Weir et al. 2011; Weir et al. 2013). En el caso de presentar un dolor moderado, pero con limitación de la movilidad, se desarrollará un trabajo de fisioterapia con readaptación físico-deportiva, mediando la movilidad de cadera y flexibilidad de los músculos periarticulares mediante la combinación de diversas técnicas (Thorborg, Serner, Petersen, Madsen, Magnusson, \& Holmich, 2011). 


\section{Conclusión}

Existe gran controversia a nivel conceptual en la patología de dolor inguinal referido al deporte. La presencia de múltiples términos en la bibliografía hace necesario la unificación de los conceptos utilizados, siendo quizás lo más adecuado la utilización del concepto de dolor inguinal. Existe una gran cantidad de causas, factores de riesgo y diagnósticos, por lo que se presenta como una patología de especial complejidad. Entre los muchos posibles factores de riesgo estudia- dos, la presencia de lesiones previas parece ser el mas claro. En muchos existe gran controversia, en especial los que hacen referencia a la funcionalidad, como la movilidad, la fuerza y el desequilibrio muscular. En la literatura científica, las intervenciones mediante ejercicio parecen ser efectivas para la recuperación y prevención del dolor inguinal en jugadores de fútbol, pero se necesitan más estudios con mayor calidad metodológica que demuestren la eficacia del tratamiento activo con diferentes propuestas metodológicas, evaluación y comparación de sus resultados.

\section{B I B LIOGRAFÍA}

Al Attar, W. S. A., Soomro, N., Pappas, E., Sinclair, P. J., \& Sanders, R. H. (2016). How Effective are F-MARC Injury Prevention Programs for Soccer Players? A Systematic Review and Meta-Analysis. Sports Medicine, 46(2), 205-217. doi:10.1007/s40279-015-0404-x

Almeida, M. O., Silva, B. N. G., Andriolo, R. B., Atallah, A. N., \& Peccin, M. S. (2013). Conservative interventions for treating exerciserelated musculotendinous, ligamentous and osseous groin pain. The Cochrane Database of Systematic Reviews, 6(1), CD009565. doi:10.1002/14651858.CD009565.pub2

Barengo, N. C., Meneses-Echávez, J. F., Ramirez-Velez, R., Cohen, D. D., Tovar, G., \& Bautista, J. E. C. (2014). The impact of the FIFA 11+ training program on injury prevention in football players: a systematic review. International Journal of Environmental Research and Public Health, 11(11), 11986-12000. doi:10.3390/ijerph111111986

Barrett, S., Midgley, A., Reeves, M., Joel, T., Franklin, E., Heyworth, R., ... Lovell, R. (2016). The within-match patterns of locomotor efficiency during professional soccer match play: Implications for injury risk? Journal of Science and Medicine in Sport, 19(10), 810-815 doi:10.1016/j.jsams.2015.12.514

Bastos, F. N., Vanderlei, F. M., Carlos, L., Vanderlei, M., Júnior, J. N., \& Pastre, C. M. (2013). Investigation of characteristics and risk factors of sports injuries in young soccer players: a retrospective study. International Archives of Medicine, 6(1), 14. doi:10.1186/1755-7682-6-14

Bisciotti, G. N., Auci, A., Marzo, F. Di, Galli, R., Pulici, L., Carimati, G., ... Volpi, P. (2015). Groin pain syndrome : an association of different pathologies and a case presentation. Muscles, Ligaments and Tendons Journal, 5(3), 214-222. doi:10.11138/mltj/2015.5.3.214

Bittencourt, N. F. N., Meeuwisse, W. H., Mendonça, L. D., Ocarino, J. M., \& Fonseca, S. T. (2016). Complex systems approach for sports injuries : moving from risk factor identi fi cation to injury pattern recognition - narrative review and new concept. British Journal of Sports Medicine, 50(1), 1309-1314. doi:10.1136/bjsports-2015-095850

Bizzini, M., Impellizzeri, F. M., Dvorak, J., Bortolan, L., Schena, F., Modena, R., \& Junge, A. (2013). Physiological and performance responses to the "FIFA 11+" (part 1): is it an appropriate warm-up? Journal of Sports Sciences, 31(13), 1481-1490. doi:10.1080/02640414.2013.80 2922

Bowen, L., Gross, A. S., Gimpel, M., \& Li, F. (2016). Accumulated workloads and the acute : chronic workload ratio relate to injury risk in elite youth football players. British Journal of Sports Medicine, 51(5), 452-459. doi:10.1136/bjsports-2015-095820

Branci, S., Thorborg, K., Bech, B. H., Boesen, M., Nielsen, M. B., \& Hölmich, P. (2014). MRI findings in soccer players with longstanding adductor-related groin pain and asymptomatic controls. British Journal of Sports Medicine, 49(10), 681-691. doi:10.1136/bjsports-2014-093710

Brown, C. K., Southerst, D., Cote, P., Shearer, H. M., Randhawa, K., Wong, J. J., ... Taylor-Vaisey, A. (2016). The Effectiveness of Exercise on Recovery and Clinical Outcomes in Patients With Soft Tissue Injuries of the Hip, Thigh, or Knee: A Systematic Review by the Onta- rio Protocol for Traffic Injury Management (OPTIMa) Collaboration. Journal of Manipulative and Physiological Therapeutics, 39(2), 110. doi:10.1016/j.jmpt.2016.01.003

Bruker, P., \& Khan, K. (2006). Clinical Sports Medicine. 3rd ed. Sydney: McGraw-Hill.

Casáis Martínez, L. (2008). Review of physical activity strategies to prevent sports injuries. Apunts Medicina de l" Esport (English Edition), 43(157), 30-40.

Charlton, P. C., Drew, M. K., Mentiplay, B. F., Grimaldi, A., \& Clark, R. A. (2017). Exercise Interventions for the Prevention and Treatment of Groin Pain and Injury in Athletes: A Critical and Systematic Review. Sports Medicine, 47(10), 2011-2026. doi:10.1007/s40279-0170742-y

Cheatham, S. W., Kolber, M. J., \& Shimamura, K. K. (2016). The Effectiveness of Nonoperative Rehabilitation Programs for Athletes Diagnosed With Osteitis Pubis. Journal of Sport Rehabilitation, 25(4), 399-403. doi:10.1123/jsr.2015-0016

Chena, M., Rodríguez, M. L., \& Cerezal, A. B. (2017). La prevención de lesiones en el fútbol según la interpretación de la naturaleza de las lesiones: reduccionismo vs complejidad. RED: Revista de Entrenamiento Deportivo, 31(4), 22-32.

Cohen, B., Kleinhenz, D., Schiller, J., \& Tabaddor, R. (2016). Understanding Athletic Pubalgia: A Review. Rhode Island Medical Journal, 99(10), 31-35.

Della-Villa, F., Mandelbaum, B.R., \& Lemak, L.J. (2018). The Effect of Playing Position on Injury Risk in Male Soccer Players: Systematic Review of the Literature and Risk Considerations for Each Playing Position. Am J Orthop (Belle Mead NJ), 47(10). doi:10.12788/ ajo.2018.0092.

Ehrmann, F. E., Duncan, C. S., Sindhusake, D., Franzsen, W. N., \& Greene, D. A. (2016). GPS and Injury Prevention in Professional Soccer. Journal of Strength and Conditioning Research, 30(2), 360-367. doi:10.1519/JSC.0000000000001093

Ekstrand, J., Hägglund, M., \& Waldén, M. (2011a). Epidemiology of Muscle Injuries in Professional Football (Soccer). The American Journal of Sports Medicine, 39(6), 1226-1232. doi:10.1177/0363546510395879

Ekstrand, J., Hägglund, M., \& Waldén, M. (2011b). Injury incidence and injury patterns in professional football : the UEFA injury study. British Journal of Sports Medicine, 45(7), 553-558. doi:10.1136/ bjsm.2009.060582

Ekstrand, J., \& Hilding, J. (1999). The incidence acute groin injuries in male soccer players. Scandinavian Journal of Medicine \& Science in Sports, 9(2), 98-103.

Elattar, O., Choi, H., Dills, V. D., \& Busconi, B. (2016). Groin Injuries (Athletic Pubalgia) and Return to Play. Sports Health, 8(4), 313-323. doi:10.1177/1941738116653711

Esteve, E., Rathleff, M. S., Bagur-Calafat, C., Urrutia, G., \& Thorborg, K. (2015). Prevention of groin injuries in sports: a systematic review with meta-analysis of randomised controlled trials. British Journal of Sports Medicine, 49(12), 785-791. doi:10.1136/bjsports-2014-094162 
Faude, O., Rommers, N., \& Rössler, R. (2018). Exercise-based injury prevention in football. German Journal of Exercise and Sport Research, 48(2), 157-168. doi:10.1007/s12662-018-0505-4

Gómez, P., y Ortega, J. (2013). Propuesta de control y seguimiento del proceso de readaptación funcional de una lesión de rodilla. FútbolPF: Revista de preparación física en fútbol, 7(1), 20-35.

Gomes Neto, M., Conceicao, C. S., de Lima Brasileiro, A. J. A., de Sousa, C. S., Carvalho, V. O., \& de Jesus, F. L. A. (2017). Effects of the FIFA 11 training program on injury prevention and performance in football players: a systematic review and meta-analysis. Clinical Rehabilitation, 31(5), 651-659. doi:10.1177/0269215516675906

Hägglund, M., Waldén, M., \& Ekstrand, J. (2016). Injury recurrence is lower at the highest professional football level than at national and amateur levels: does sports medicine and sports physiotherapy deliver? British Journal of Sports Medicine, 50(12), 751-758. doi:10.1136/ bjsports-2015-095951

Hägglund, M., Waldén, M., \& Ekstrand, J. (2005). Injury incidence and distribution in elite football - a prospective study of the Danish and the Swedish top divisions. Scandinavian Journal of Medicine \& Science in Sports, 15(1), 21-28.

Hammes, D., Aus der Funten, K., Kaiser, S., Frisen, E., Bizzini, M., \& Meyer, T. (2015). Injury prevention in male veteran football players - a randomised controlled trial using "FIFA 11+". Journal of Sports Sciences, 33(9), 873-881. doi:10.1080/02640414.2014.975736

Holmich, P., Thorborg, K., Dehlendorff, C., Krogsgaard, K., \& Gluud, C. (2014). Incidence and clinical presentation of groin injuries in sub-elite male soccer. British Journal of Sports Medicine, 48(16), 1245-1250. doi:10.1136/bjsports-2013-092627

Holmich, P., Thorborg, K., Nyvold, P., Klit, J., Nielsen, M. B., \& Troelsen, A. (2014). Does bony hip morphology affect the outcome of treatment for patients with adductor-related groin pain? Outcome 10 years after baseline assessment. British Journal of Sports Medicine, 48(16), 1240-1244. doi:10.1136/bjsports-2013-092478

Impellizzeri, F. M., Bizzini, M., Dvorak, J., Pellegrini, B., Schena, F., \& Junge, A. (2013). Physiological and performance responses to the FIFA 11+ (part 2): a randomised controlled trial on the training effects. Journal of Sports Sciences, 31(13), 1491-1502. doi:10.1080/ 02640414.2013 .802926

King, E., Ward, J., Small, L., Falvey, E., \& Franklyn-Miller, A. (2015). Athletic groin pain: a systematic review and meta-analysis of surgical versus physical therapy rehabilitation outcomes. British Journal of Sports Medicine, 49(22), 1447-1451. doi:10.1136/bjsports-2014-093715

Kloskowska, P., Morrissey, D., Small, C., Malliaras, P., \& Barton, C. (2016). Movement Patterns and Muscular Function Before and After Onset of Sports-Related Groin Pain: A Systematic Review with Metaanalysis. Sports Medicine, 46(12), 1847-1867. doi:10.1007/s40279016-0523-z

Langhout, R., Tak, I., Beijsterveldt, A.-M. van, Ricken, M., Weir, A., Barendrecht, M., ... Stubbe, J. (2018). Risk Factors for Groin Injury and Symptoms in Elite Level Soccer Players: A Cohort Study in the Dutch Professional Leagues. J Orthop Sports Phys Ther, 48(9), 704-712.

Leventer, L., Eek, F., Hofstetter, S., \& Lames, M. (2016). Injury Patterns among Elite Football Players: A Media-based Analysis over 6 Seasons with Emphasis on Playing Position. International Journal of Sports Medicine, 37(11), 898-908. doi:10.1055/s-0042-108201

Malone, S., Owen, A., Mendes, B., Hughes, B., Collins, K., \& Gabbett, T. J. (2018). High-speed running and sprinting as an injury risk factor in soccer: Can well-developed physical qualities reduce the risk? Journal of Science and Medicine in Sport, 21(3), 257-262. doi:10.1016/j. jsams.2017.05.016

Malone, S., Owen, A., Newton, M., Mendes, B., Collins, K.D., Gabbett, T.J. (2017). The acute: chonic workload ratio in relation to injury risk in professional soccer. J Sci Med Sport, 20(6), 561-565.

Mayo, M., Seijas, R., \& Álvarez, P. (2014). Structured neuromuscular warm-up for injury prevention in young elite football players. Rev Esp Cir Ortop Traumatol, 58(6), 336-342. doi:10.1016/j.recot.2014.05.008

McCall, A., Carling, C., Davison, M., Nedelec, M., Le Gall, F., Berthoin, S., \& Dupont, G. (2015). Injury risk factors, screening tests and preven- tative strategies: a systematic review of the evidence that underpins the perceptions and practices of 44 football (soccer) teams from various premier leagues. British Journal of Sports Medicine, 49(9), 583589. doi:10.1136/bjsports-2014-094104

Meyers, W. C., Yoo, E., \& Horner, M. (2008). Understanding "sports hernia " (athletic pubalgia) - The anatomic and pathophysiologic basis for abdominal and groin pain in athletes. Operative Techniques in Sports Medicine, 20(1), 33-45.

Mosler, A. B., Agricola, R., Weir, A., Holmich, P., \& Crossley, K. M. (2015). Which factors differentiate athletes with hip/groin pain from those without? A systematic review with meta-analysis. British Journal of Sports Medicine, 49(12), 810. doi:10.1136/bjsports-2015-094602

Mosler A.B., Weir, A., Eirale, C., Farooq, A., Thorborg, K., Whiteley, R., Holmich, P., \& Crossley, K.M. (2018). Epidemiology of time loss groin injuries in a men's professional football league: a 2-year prospective study of 17 clubs and 606 players. Br J Sports Med, 52(5), 292-297. doi:10.1136/bjsports-2016-097277

Naranjo, T., Bayo, T., Fernández, G., \& Salas, M. (2012). Pubalgia: diagnóstico diferencial. Revista Internacional de Medicina y Ciencias de La Actividad Física y El Deporte, 12(48), 757-769

Nevin, F., \& Delahunt, E. (2014). Adductor squeeze test values and hip joint range of motion in Gaelic football athletes with longstanding groin pain. Journal of Science and Medicine in Sport, 17(2), 155-159. doi:10.1016/j.jsams.2013.04.008

Noya, J., \& Sillero, M. (2012a). Epidmeiología de las lesiones en el fútbol profesional español en la temporada 2008-2009. Archivos de Medicina Del Deporte, XXIX(150), 750-766.

Noya, J., \& Sillero, M. (2012b). Incidencia lesional en el fútbol profesional español a lo largo de una temporada: días de baja por lesión. Apunts. Medicina de l'Esport, 47(176), 115-123.

Orchard, J. W. (2015). Men at higher risk of groin injuries in elite team sports: a systematic review. British Journal of Sports Medicine, 49(12), 798-802. doi:10.1136/bjsports-2014-094272

Owen, A. L., Wong, D. P., Dellal, A., Paul, D. J., Orhant, E., \& Collie, S. (2013). Effect of an injury prevention program on muscle injuries in elite professional soccer. Journal of Strength and Conditioning Research, 27(12), 3275-3285. doi:10.1519/JSC.0b013e318290cb3a

Panasiuk, A. (2009). Estudio retrospectivo sobre la prevalencia de las principales lesiones de los futbolistas profesionales en el Uruguay, abril 1997 - mayo 2007. Revista AKD, 1(1) 8-10.

Polinder, S., Haagsma, J., Panneman, M., Scholten, A., Brugmans, M., \& Van Beeck, E. (2016). The economic burden of injury: Health care and productivity costs of injuries in the Netherlands. Accident Analysis \& Prevention, 93(1), 92-100. doi:10.1016/j.aap.2016.04.003

Quatman, C., Quatman, C., \& Hewet, T. (2009). Prediction and prevention of musculoskeletal injury: a paradigm shift in methodology. British Journal of Sports Medicine, 43(14), 1100-1107. doi:10.1136/ bjsm.2009.065482.

Robertson, B. A., Barker, P. J., Fahrer, M., \& Schache, A. G. (2009). Pathogenesis and Clinical Management of Chronic Groin Pain in Athletes The Anatomy of the Pubic Region Revisited Implications for the Pathogenesis and Clinical Management of Chronic Groin Pain in Athletes. Sports Medicine, 39(3), 225-234. doi:10.2165/00007256200939030-00004

Rodríguez, C., Miguel, A., Lima, H., \& Heinrichs, K. (2001). Osteitis Pubis Syndrome in the Professional Soccer Athlete: A Case Report. Journal of Athletic Training, 36(4), 437-440.

Ryan, J., DeBurca, N., \& Mc Creesh, K. (2014). Risk factors for groin/ hip injuries in field-based sports: a systematic review. British Journal of Sports Medicine, 48(14), 1089-1096. doi:10.1136/bjsports-2013-092263

Sadigursky, D., Braid, J. A., De Lira, D. N. L., Machado, B. A. B., Carneiro, R. J. F., \& Colavolpe, P. O. (2017). The FIFA 11+ injury prevention program for soccer players: a systematic review. BMC Sports Science, Medicine \& Rehabilitation, 9(1), 18. doi:10.1186/s13102-017-0083-z

Salces, N., Carmona, G., Marco, G., Urdiales, M., Quintana, S., Salces, N., ... Sillero-quintana, M. (2014). Epidemiology of injuries in First Division Spanish football. Journal of Sports Sciences, 32(13), 1263-1270.

Schoberl, M., Prantl, L., Loose, O., Zellner, J., Angele, P., Zeman, F., ... Krutsch, W. (2017). Non-surgical treatment of pubic overload 
and groin pain in amateur football players: a prospective doubleblinded randomised controlled study. Knee Surgery, Sports Traumatology, Arthroscopy: Official Journal of the ESSKA, 25(6), 1958-1966. doi:10.1007/s00167-017-4423-z

Silvers-Granelli, H., Mandelbaum, B., Adeniji, O., Insler, S., Bizzini, M., Pohlig, R., ... Dvorak, J. (2015). Efficacy of the FIFA 11+ Injury Prevention Program in the Collegiate Male Soccer Player. The American Journal of Sports Medicine, 43(11), 2628-2637. doi:10.1177/0363546515602009

Tak, I., Engelaar, L., Gouttebarge, V., Barendrecht, M., Van den Heuvel, S., Kerkhoffs, G., ... Weir, A. (2017). Is lower hip range of motion a risk factor for groin pain in athletes? A systematic review with clinical applications. British Journal of Sports Medicine, 51(22), 1611-1621. doi:10.1136/bjsports-2016-096619

Thomson, A., Whiteley, R., \& Bleakley, C. (2015). Higher shoe-surface interaction is associated with doubling of lower extremity injury risk in football codes: a systematic review and meta-analysis. British Journal of Sports Medicine, 49(19), 1245-1252. doi:10.1136/bjsports-2014-094478

Thorborg, K., Krommes, K. K., Esteve, E., Clausen, M. B., Bartels, E. M., \& Rathleff, M. S. (2017). Effect of specific exercise-based football injury prevention programmes on the overall injury rate in football: a systematic review and meta-analysis of the FIFA 11 and 11+ programmes. British Journal of Sports Medicine, 51(7), 562-571. doi:10.1136/ bjsports-2016-097066

Thorborg, K., Serner, A., Petersen, J., Madsen, T.M., Magnusson, P., Holmich, P. (2011). Hip adduction and abduction strength profiles in elite soccer players: implications for clinical evaluation of hip adductor muscle recovery after injury. The American Journal of Sports Medicine, 39(1), 121-126. doi:10.1177/0363546510378081

Topol, G.A., Reeves, K.D., Hassanein K.M. (2005). Efficacy of dextrose prolotherapy in elite male kicking-sport athletes with chronic groin pain. Archives of Physical Medicine and Rehabilitation, 86(4), 697-702. doi:10.1016/j.apmr.2004.10.007

Verrall, G. M., Slavotinek, J. P., Fon, G. T., \& Barnes, P. G. (2007). Outcome of Conservative Management of Athletic Chronic Groin Injury Diagnosed as Pubic Bone Stress Injury. The American Journal of Sports Medicine, 35(3), 467-474. doi:10.1177/0363546506295180

Walde, M., \& Ekstrand, J. (2009). UEFA injury study: a prospective study of hip and groin injuries in professional football over seven consecutive seasons. Br J Sports Med, 43(13), 1036-1040. doi:10.1136/ bjsm.2009.066944

Wald, M., Hagglund, M., \& Ekstrand, J. (2005). UEFA Champions League study: a prospective study of injuries in professional football during the 2001-2002 season. Br J Sports Med, 39(8), 542-546. doi:10.1136/ bjsm.2004.014571

Weir, A., Brukner, P., Delahunt, E., Ekstrand, J., Griffin, D., Khan, K. M. ... Holmich, P. (2015). Doha agreement meeting on terminology and definitions in groin pain in athletes. British Journal of Sports Medicine, 49(12), 768-774. doi:10.1136/bjsports-2015-094869

Weir, A., Jansen, N., Dijkstra, S., Backx, F., \& Tol, J. (2013). Manual or exercise therapy for long-standing adductor-related groin pain: Midterm follow-up of a randomised controlled clinical trial. European Journal of Sports Medicine, 1(1), 39-46.

Weir, A., Jansen, J. A. C. G., Van de Port, I. G. L., Van de Sande, H. B. A., Tol, J. L., \& Backx, F. J. G. (2011). Manual or exercise therapy for long-standing adductor-related groin pain: a randomised controlled clinical trial. Manual therapy, 16(2), 148-154. doi:10.1016/j. math.2010.09.001

Williams, J. H., Akogyrem, E., \& Williams, J. R. (2013). A Meta-Analysis of Soccer Injuries on Artificial Turf and Natural Grass. Journal of Sports Medicine, 1(1), 380523. doi:10.1155/2013/380523 